\title{
LOS VAIVENES DE \\ LA POLITICA SOCIAL \\ DEL ESTADO: \\ el caso de los hogares infantiles del ICBF
}

\author{
Por: Maria Cristina Salazar*
}

\section{NACEN LOS HOGARES INFANTILES}

En Colombia, antes de los años 70 no existían modelos estatales de atención y educación pre-escolar para la población menor de 7 años, por lo cual una misión de la Organización Mundial de la Salud, en 1970, recomendó al gobierno el establecimiento en el Instituto Colombiano de Bienestar Familiar (ICBF) de un programa dirigido a la atención "integral" de los niños en edad pre-escolar, que a la vez promoviera "la estabilidad de la familia". El programa contemplaba la coordinación de los servicios de nutrición, salud, educación, promoción social, y protección legal, y debía dirigirse a los sectores más pobres de ciudades y campos.

Los Hogares Infantiles, antes conocidos como CAIPs (Centros de Atención Integral al Preescolar), fueron concebidos como centros donde se cuidaría a los niños menores de 7 años, cuyas madres estuvieran trabajando fuera de su propio hogar. En ellos se iniciaría un proceso de educación pre-escolar, y sus sedes servirían como sitios de reunión para las comunidades con el fin de que estas emprendieran acciones y proyectos de desarrollo social. Para llevar a cabo estas ideas, se propuso la coordinación de las agencias de bienestar de los sectores público y privado, y se hizo énfasis en el logro del mayor cubrimiento posible de la población de bajos recursos. Siguiendo las recomendaciones de la OMS, el gobierno lanzó un ambicioso programa de atención integral para la población pre-escolar, por medio de la ley 27 de 1974 que creó los CAIPs --hoy conocidos como Hogares Infantiles-. Consagró la obligación de las empresas públicas y privadas de aportar el $2 \%$ de su nómina mensual al ICBF con el fin de financiar los nuevos Centros $\left({ }^{19}\right)$.

Antes de la expedición de la ley 27, las empresas que tenían más de 50 trabajadoras estaban legalmente obligadas a establecer centros de cuidado diurno para los hijos de las mismas. Este sistema se basaba en la idea, aún prevaleciente en muchos círculos, de

\footnotetext{
* Socióloga. Profesora de la Universidad Nacional de Colombia. Este artículo se basa en investigaciones realizadas sobre Hogares Infantiles en el sur-oriente de Bogotá, de 1984 a 1986 (ver Salazar, 1986).

${ }^{19}$ Varias informaciones coinciden en señalar que el dinero recaudado - que llega a más de 10.000 millones de pesos en 1986, pero que desde 1979 pasó a formar parte del presupuesto general del ICBF--, responde hoy en día por la casi totalidad de egresos del ICBF, por lo cual disminuye considerablemente los recursos que antes eran asignados a los Hogares Infantiles. Entrevista al director general del ICBF. El Tiempo junio 21, 1984. Entrevistas a funcionarios del ICBF, Sede Regional de Bogotá, 1986, hechas por la autora.
} 
que no corresponde al estado la atención de los niños menores de 7 años. Se asume que la familia, en especial la madre, son quienes deben cuidar del niño pequeño. Con la ley 27, aquellas empresas que antes tenían sus propias guarderías, empezaron a enviar a los niños de sus empleadas y obreras a los Centros del ICBF. Es así como existen en la actualidad algunos Hogares Infantiles casi exclusivamente dedicados a atender a los niños de trabajadoras de empresas del sector moderno de la economía, lo que significó un simple traslado de niños de las guarderías de las empresas privadas a las nuevas creadas por el Estado, con considerables costos para el presupuesto del ICBF y posibles economías para las empresas beneficiadas.

La ley 27 se aprobó sin mayor discusión pública, aunque se expresaron distintas opiniones sobre la población que habría de recibir los beneficios del programa. Tanto el gobierno como las centrales obreras, se inclinaron a favorecer a los hijos de los trabajadores; por lo mismo, la ley se aprobó para ellos, dejando por fuera a los niños de padres desempleados. Sin embargo, posteriormente, en especial por presión del Departamento Nacional de Planeación, se logró la modificación de la ley para incluirlos, aunque en la práctica se ha comprobado la tendencia contraria (Bonilla 1981:29). El propósito de favorecer a la madre trabajadora, loable es cierto, dejó por fuera a una inmensa población de madres en busca de trabajo, o que se desempeñan esporádicamente como empleadas del servicio doméstico, excluyendo así a muchos niños en urgente necesidad de atención. Algunas evaluaciones de los Hogares Infantiles presentan evidencia para confirmar que se otorga preferencia a las madres del sector moderno de la economía (Mora y Mantilla 1980). El mismo ICBF (Sede Regional Bogotá 1985) señaló que "la población con mayor índice de desprotección no recibe la atención de los Hogares Infantiles" y afirmó la urgencia de llegar a los sectores más desprotegidos, después de un censo de los Hogares en Bogotá.

\section{LA PLANEACION Y LOS HOGARES INFANTILES}

La planeación corresponde en Colombia al Congreso de la República, con delegación en los Ministerios, el DNP y la Dirección General del Presupuesto. El ICBF, como instituto descentralizado, actúa en la ejecución de los planes contemplados en las políticas globales. Su principal función es "ejecutar las políticas del gobierno nacional en materia de fortalecimiento de la familia y protección al menor de edad" (Decreto 334 de 1980); su Junta Directiva formula la política general del Instituto, de acuerdo con el Ministerio de Salud, el DNP, los planes sectoriales y el Plan Nacional de Desarrollo (art.26, ley 7 de 1979). La misma Junta debe "determinar la cobertura progresiva de los Hogares Infantiles" (ibid).

Las complejidades de un proceso de planeación como el que resulta de la legislación anterior saltan a la vista: es casi imposible lograr participación de los niveles regionales o locales en el proceso, dando como resultado la verticalidad que lo caracteriza, pase a la existencia de numerosos documentos del ICBF que enfatizan la planeación como proceso de abajo hacia arriba. Por otra parte, la ausencia de investigación y de una reflexión permanente en las Unidades Regionales y Locales del ICBF sobre sus áreas de influencia y las condiciones de las comunidades que reciben los servicios del Instituto, hace que rara vez se tengan en cuenta las opiniones de estas últimas.

En el caso de los Hogares Infantiles, estas limitaciones se manifiestan de diversos modos. Por ejemplo, la decisión de establecer Hogares en lugares específicos, no es consultada con las comunidades, como tampoco lo es la delegación de la administración de cada Hogar a grupos diversos. Así se contradicen las declaraciones oficiales sobre 
participación de los grupos de base. Menos aún se consultan decisiones tales como las relativamente recientes sobre la eliminación gradual de los Hogares, con el fin de reemplazarlos por modalidades de atención que sólo tienen en cuenta la ampliación de las coberturas y la reducción de costos, sin pensar en la calidad del servicio.

\section{EXTENSION DE LOS HOGARES INFANTILES}

En el país se han establecido, desde 1975, cerca de 1.500 Hogares, la mayor parte de ellos en las capitales departamentales (el $90 \%$ en áreas urbanas). El número de niños atendidos ha ido en aumento: de 72.000 en 1980, a más de 200.000 en 1984 (ICBF Informes 1982, 1984). Pero a pesar de ello, la cobertura de la población de bajos ingresos, menor de 7 años, es baja: entre el uno y el cinco por ciento. En Bogotá, se estimó esta proporción en el 1.5\% en 1981 (Bonilla 1981); en 1984, el DNP señaló que la cobertura nacional era del 2.8\% (Puyana, 1984), y en 1985 la Sede Regional de Bogotá afirmó que sólo el $5 \%$ de los niños bogotanos en "alto riesgo" era atendido en los Hogares del ICBF.

Hasta 1986, cuando se realizaron las investigaciones en que se basa este artículo, no parecía haber posibilidades de ampliación de las coberturas, pero sorpresivamente, a principios de 1987, se conoció el plan de los Hogares Comunitarios que busca atender a un millón 500 mil niños en el país, mediante la creación de 100.000 Hogares, dirigidos por madres de los sectores populares, a quienes se otorgan honorarios de $\$ 15$ mil mensuales y un aporte para la alimentación de cada niño. Este plan aún no ha sido evaluado; por el momento puede decirse que mediante esos Hogares (se han establecido hasta la fecha 8.033 en todo el país) se podrán abrir posibilidades de organización y discusión en las comunidades acerca de la niñez y de la responsabilidad del Estado al respecto. Pero subsiste la duda acerca de la calidad que pueda tener esa atención debido a los escasos recursos que se asignan al plan, y por lo mismo, puede servir para que se continúe ampliando la brecha entre los cuidados y la educación pre-escolar que reciben los niños de los sectores privilegiados, una minoría, y los niños de los sectores populares. En editorial, El Tiempo (diciembre 4 de 1987), manifestaba las dudas que le suscitaban las palabras del director del ICBF respecto al "éxito" del programa, ya que había ocurrido una manifestación de protesta frente a la sede nacional del Instituto, precisamente demandando mejor atención para los niños de los Hogares Infantiles.

\section{LA ADMIINISTRACION DE LOS HOGARES INFANTILES}

El Instituto optó por delegar la administración de cada Hogar Infantil en asociaciones de carácter cívico sin ánimo de lucro, medida que busca evitar el aumento de la ya crecida planta de funcionarios y el costo de la nómina. Los Hogares Infantiles en 1984 tenían cerca de 15 mil empleados en el país. La medida naturalmente impide, además, la organización sindical de los empleados y jardineros, ya que todos ellos tienen un patrón determinado y diferente al estado. En 1986 todos los Hogares Infantiles en el país tenían una administración delegada; principalmente en asociaciones de carácter religioso y cívico que se encargan de lo relacionado con las obligaciones laborales de su personal.

Estas asociaciones imponen algunas de sus normas en la práctica, tanto en lo relativo a la atención y educación pre-escolar de los niños como a las condiciones laborales de los jardineros y empleados de los Hogares. En Hogares Infantiles de Bogotá, observamos que influyen en el contenido de la educación preescolar y en la pedagogía, en las relaciones con los hogares de los niños e igualmente en las relaciones que se establecen entre los jardineros y los niños. En otro escrito señalábamos que así se sigue el modelo 
de descentralización que ocurre en la industria, con fines semejantes: se evade el control estatal o por lo menos se hace más difícil, se toma ventaja para pagar bajos salarios, para contratar personal no calificado, se evita el pago de prestaciones sociales, etc. $\left({ }^{20}\right)$.

\section{OBJETIVOS DE LOS HOGARES INFANTILES}

Al comparar los objetivos iniciales de los Hogares con los servicios que prestan, se comprueba una reducción dramática. Hasta el punto de que en 1984 se afirmaba que los Hogares debían cerrarse -como en efecto se ha venido haciendo en todo el paísaduciendo restricciones presupuéstales que nunca faltan. $\left({ }^{21}\right)$. Conceptos que dieron origen al programa -atención integral, servicios integrados, desarrollo y participación comunitaria- se repiten en documentos y leyes, y como es usual en las declaraciones oficiales, las metas señaladas iban mucho más allá de lo que el Instituto era capaz de realizar. Por ejemplo, que los Hogares "podrán elevar los niveles de vida del niño y de su respectiva comunidad", y detrás de ésta y otras declaraciones encontramos una concepción que puede tildarse de "mágica", puesto que se describen programas vagos y confusos, por ejemplo el de la educación en familia, como la panacea de los problemas que afectan a la niñez de la población pobre. Algunas declaraciones oficiales aterrizan en la realidad y son más concretas, aunque rara vez se cumplen; por ejemplo las especificaciones sobre la atención integral.

\section{LA CAPACITACION DE LOS JARDINEROS}

Otro aspecto de la educación pre-escolar que se ha descuidado en los Hogares es el relacionado con la capacitación de los (las) jardineros (as). Estos tienen niveles de educación que por lo general no superan el nivel secundario incompleto; muchos carecen por completo de capacitación en educación preescolar y los que han logrado ingresar a los institutos "universitarios" (muchos de ellos piratas> de educación pre-escolar, reciben una capacitación deficiente. Esta situación se refleja en la falta de interés de muchos jardineros (as) por las actividades propiamente educativas y de desarrollo de las capacidades intelectuales de los niños. A los niños se les cuida, se les alimenta adecuadamente, y también se les entretiene de diversas maneras; lo esencial parece ser el simple cuidado de los niños. No descubrimos, en largas y repetidas observaciones de varios Hogares Infantiles, intentos por reflexionar sobre el desarrollo intelectual y afectivo de los niños. Vimos, sí, gran dedicación y afecto por los niños, y a veces pensábamos, las jardineras son "mujeres-niñas", casi jugando a ser mamás.

\section{EVALUACIONES DE LOS HOGARES INFANTILES}

Lo primero que hay que afirmar es que el establecimiento de Hogares Infantiles para el sector de la población más pobre como programa estatal es importante como un paso para que el estado cumpla sus responsabilidades frente a niños que de otro modo estarían abandonados o encerrados mientras sus madres salen a trabajar. También puede afirmarse que los Hogares tienen la posibilidad de convertirse en entidades innovadores dentro de las comunidades, de gran alcance, en relación con los servicios que podrían suministrar enfatizando su carácter comunitario como lo establecen las normas correspondientes.

\footnotetext{
${ }^{20}$ Se han generado distintos problemas relacionados con estas disposiciones como se desprende de declaraciones oficiales que reiteradamente precisan que esos trabajadores no son empleados del ICBF (véase por ejemplo la carta del director general a los directores de las Sedes Regionales, octubre 3 de 1985)

${ }^{21}$ El subdirector del Departamento de Nutrición del ICBF señaló: "No hay dinero suficiente para edificaciones de CAIPs, ni para su mantenimiento, aún cuando existan las sedes para su funcionamiento'. El Tiempo febrero 6 de 1984.
} 
Pero lo que pasa es que no se cumplen las normas en aspectos esenciales, tales como la colaboración entre las distintas instituciones, la contratación de personal competente, la capacitación de éste, la asignación de responsabilidades a distintos sectores de la comunidad, etc. Y el vicio del clientelismo no falta, por lo cual se hacen nombramientos en favor de clientelas políticas determinadas, o de personal incompetente para ahorrar costos, como ocurre entre las entidades administradoras, en especial las de carácter religioso.

Los contenidos de las acciones se han limitado y hay un gran desfase entre lo propuesto en los documentos y lo efectivamente llevado a cabo. Casi que sólo se atiende a la nutrición y al cuidado físico de los niños, dejando de últimos los contenidos y procesos educativos y comunitarios. La integración de los servicios deja mucho que desear: no se están acometiendo procesos reales de integración, tal vez por fallas en el énfasis dado al factor educativo como lazo de unión entre los distintos elementos programáticos. Deja mucho que desear la asistencia técnica por parte del ICBF a los Hogares, tanto a nivel regional como zonal.

Además, desde 1980 aproximadamente, en el ICBF ha prosperado una corriente que favorece el asignar los aspectos de educación de los Hogares al Ministerio de Educación. Varios funcionarios nos comentaron en el curso de la investigación a que nos hemos referido, que les parecía errónea la perspectiva inicial de los CAIPs en cuanto a incluir la educación pre-escolar en los Hogares. Según esta corriente, el ICBF debería atender sólo a los niños menores de 5 años y únicamente en lo referente al cuidado físico y nutricional, dejando por fuera a los niños de 6 y 7 años y todo lo relativo al proceso de educación preescolar, que estaría a cargo del Ministerio de Educación. Tal vez sea una consecuencia de estas concepciones el que no se dé suficiente importancia al elemento educacional en los Hogares Infantiles, a pesar de ser tan importante para el desarrollo de los niños y para evitar, o por lo menos disminuir, las desigualdades educativas entre el sector pobre y los sectores privilegiados del país.

Dentro del mismo ICBF no han faltado intentos críticos de evaluación de los Hogares. En 1977 se hicieron críticas al alto costo de las inversiones y del funcionamiento de los Hogares, y se señaló su escasa influencia comunitaria. Un sector empresarial llegó a proponer que se regresara a la situación anterior a la ley 27, es decir, que las empresas volvieran a establecer guarderías, desligándose de los CAIPs estatales.

La crisis del modelo estatal provenía de los mismos supuestos sobre los cuales se basaba, ya que se había buscado implantar en ellos el modelo de jardín infantil propio del sector privado. Este modelo podía adaptarse para la población de ingresos medios y altos, en donde existe una mayor continuidad entre el medio familiar y el medio educacional del centro pre-escolar. Pero esta continuidad no siempre se expresa en las comunidades de los sectores pobres a los cuales se dirigía el programa del ICBF, y en los que existen problemas graves de salud y desnutrición.

Otro aspecto que se señala en las evaluaciones es el relacionado con el origen de las jardineras: en el sector privado, ellas provienen de los mismos medios a que pertenecen los niños que cuidan y educan, y además pueden tener diversos vínculos con las familias de los niños. En los Hogares del ICBF, las jardineras pueden provenir de diferentes medios sociales, y sobre todo cuando ellas provienen de medios más altos, se presentan problemas en el sentido de considerar inferiores a los niños de los Hogares. Esto da como resultado un énfasis en actitudes asistencialistas: una de ellas se expresa en la búsqueda de la reducción de costos y la ampliación de las coberturas, -lo que sigue ocurriendo en 
1987-, y otra es recurrir a la tecnología, utilizando por ejemplo técnicas que mejoren los nutrientes del niño, lo que significa "socorrerlo", pero no enfocando las acciones institucionales hacía el cambio de las condiciones en que viven los niños de los sectores pobres. Estas actitudes se vieron por ejemplo en un Hogar Infantil ubicado en un sector chircalero en el suroriente de Bogotá, a cargo de una fundación, patrocinada por la familia propietaria de los terrenos de los chircales.

Se señalaba también que los padres de familia eran vistos más como usuarios de un servicio que como partícipes en el proceso de educación pre-escolar y comunitario de los Hogares. Por eso mismo, se explicaba su pasividad (Velásquez y Concha, 1981>. La delegación de la administración de los Hogares en asociaciones del sector privado se considera como un retroceso: es colocar la educación pre-escolar en manos de sectores que tradicionalmente han tenido el control de la educación en el país.

Desde 1977 se han hecho intentos de modificación de los supuestos básicos del programa de Hogares Infantiles, dentro del ICBF. Se ha visto la necesidad de contar con un movimiento social y cultural amplio, promovido por las comunidades en que se han establecido los Hogares. Se discute la importancia de establecer nuevos medios de comunicación con los padres de familia, de lograr cambios en las concepciones corrientes acerca de la educación de los niños pequeños, cambios que deben referirse a ideas vigentes sobre autoridad, sanciones y premios, como elementos del proceso de educación. Igualmente se analiza la necesidad de redefinir el proceso de participación comunitaria y de rescatar los valores comunitarios de solidaridad frente a la solución de problemas comunes. Todo ello llevó a una mayor claridad dentro del ICBF respecto a lograr que los Hogares lleguen a pertenecer a las mismas comunidades.

\section{EL CASO DE ACAIPA (ASOCIACION DE CAIP EN ANTIOQUIA)}

Merece ser destacado este modelo de atención a la niñez en edad pre-escolar, en el que el ICBF se asoció con los jardineros de 34 Hogares Infantiles ubicados en el campo, y con los padres de familia de los niños para promover un cambio en la relación maestroalumno, formando a los jardineros como promotores del desarrollo. Para ello se le asignan a cada jardinero tareas concretas de promoción comunitaria. A los padres de familia se les asignan tareas de carácter administrativo que incluyen por ejemplo la selección de jardineros, aspectos económicos, nutrición de los niños, etc.

Se desarrollan programas de formación pedagógica para jardineros y padres de familia, convirtiéndose todos en co-educadores dentro de su respectiva comunidad. Este modelo podría ser objeto de análisis en el ICBF para ver la manera de extenderlo a Hogares Infantiles de las ciudades.

\section{CAMBIOS EN LAS POLITICAS DE ATENCION A LA NIÑEZ}

En 1982, con el cambio de gobierno, se decide "reorientar" el programa de Hogares Infantiles, enfatizando principios de educación permanente al niño menor de 7 años y de participación comunitaria (ICBF 1983). Por educación permanente se entiende la continuidad de la educación que recibe el niño en su hogar, en su vecindario y en los Hogares, así como la utilización de los recursos educativos de las comunidades. Por participación comunitaria se entiende la capacidad de la comunidad para organizarse y hallar soluciones alternativas a sus problemas, con apoyo de recursos estatales. 
El Instituto buscó convertir los Hogares Infantiles en Hogares Alternos, es decir, con atención de media jornada únicamente. Naturalmente esto no correspondía a las necesidades reales de la población, puesto que las mujeres, madres de los niños, trabajan en jornadas completas que se prolongan por las distancias en las grandes ciudades. Pero la preocupación fundamental del Instituto era ampliar la cobertura, sin darle la importancia debida al cómo hacerlo. Se desconocía en esta propuesta la dificultad para los jardineros de atender en doble jornada a niños pequeños, en grupos por lo general de 30 a 40 niños. Pedagógicamente parecía un disparate intentarlo.

Se plantearon otras dos alternativas aún menos realistas que la anterior: lograr la vinculación ad honorem de los adultos del barrio, las madres en especial, para atender a grupos de 10 a 15 niños de 3 a 6 años de edad. A esas trabajadoras voluntarias se les daría una capacitación a cargo de las jardineras de los Hogares Infantiles ya establecidos (desconociendo el escaso nivel educativo de muchas de ellas), y se adaptarían espacios en las viviendas de las voluntarias para recibir a los niños (también desconociendo la precariedad de esas viviendas en los sectores pobres de las ciudades). Peor aún, se pensaba que la atención a los niños podía darse apenas durante algunas horas semanales, en contra de las necesidades reales de las madres.

La tercera alternativa propuesta por el ICBF consistía en que otros niños, mayores de 7 años, se encargaran de los niños más pequeños, también por turnos, durante algunas horas diarias, lo cual evidentemente no beneficiaba ni a las madres, ni por supuesto a los niños menores ni mayores.

De diferentes maneras, el ICBF ha venido auspiciando estas iniciativas. La que cursa actualmente es la segunda, sólo que con honorarios para las madres y con algunos recursos para la remodelación de espacios en sus viviendas. Pude conocer programas a cargo de distintos niños de corta edad en distintas ciudades del país, en una de las cuales una niña de unos 10 me dijo: “¿Por qué no nos mandan una maestra de verdad para 'mis' niños que son tan inteligentes?"

En las tres modalidades, prácticamente desaparecen los elementos educacionales y se disminuyen sustancialmente los beneficios nutricionales que hasta ahora eran bastante satisfactorios en los Hogares Infantiles. El papel del ICBF se reduce considerablemente: se limita a algunos cursos de entrenamiento a los padres de familia para que produzcan materiales pedagógicos con elementos de desecho, a la dotación de unos pocos muebles, utensilios y un refrigerio para los niños así "atendidos". Es más bien un escándalo que se hagan programas estatales en esta forma, que sustentan la idea de tratar a los sectores pobres con medios pobres.

Pero en el Instituto se considera que de esta manera se obtendrán recursos para ampliar la cobertura de los programas, duplicarla o centuplicarla, no importa cómo. En 1985 decíamos que estas alternativas empezarían a llevarse a cabo, a pesar de que significaban un retroceso considerable en las políticas estatales de atención y protección a la niñez colombiana. Hoy tenemos que decir que infortunadamente, por factores diversos, entre ellos los altos costos de los primeros Hogares Infantiles, el aumento de la burocracia dentro del ICBF, la crisis fiscal del gobierno, las presiones del Fondo Monetario Internacional para la reducción de gastos en el sector social, ocurre una reducción progresiva de los aportes nacionales al ICBF y la adopción de políticas de autofinanciación. Estas corrientes hacen que los Hogares Infantiles pasen a un segundo plano, y fortalecen la tendencia a desmontarlos, estableciendo modalidades que obligan a 
las familias y comunidades pobres del país a asumir la responsabilidad casi total de la protección y atención a los niños.

\section{BIBLIOGRAFIA}

BONILLA, E. La madre trabajadora. CEDE. Universidad de los Andes. Bogota, 1981.

ICBF. Subdirección Técnica de Protección. Modalidades de Atención al Niño con participación de la familia y la comunidad. Documento de referencia para la reorganización del Proyecto Hogares Infantiles. Bogotá, 1983.

ICBF. Sede Regional de Bogotá, 1985. Censo de usuarios de Hogares Infantiles Comunitarios y Vecinales del ICBF en Bogotá. Bogotá.

MORA, A. y Mantilla, M. Los Centros de Atención Integral al Pre-escolar como respuesta a un problema socioeconómico en el área metropolitana de Bogotá. Universidad Nacional de Colombia. Bogotá, 1980.

PUYANA, Y. Informe sobre la familia en Colombia. Departamento Nacional de Planeación. Bogotá, 1984.

SALAZAR, M.C. Políticas estatales y el bienestar de la niñez. Los Hogares Infantiles del ICBF en Bogotá. Universidad Nacional de Colombia. Bogotá.

VELASQUEZ, E. y Concha. Modelos alternativos. ICBF. Bogotá, 1981. 